\title{
Pattern Recognition of Partial Discharge by Using Scale parameters-Energy Entropy Characteristic Pairs
}

\author{
Liu Dongchao*, Xiong Hui, Zhu Xiaotong, Xu Lei \\ NR Electric Co. Ltd. Nanjing, 211102, China
}

\begin{abstract}
In this paper, the complex wavelet transform (CWT) was used to process the ultra-high frequency partial discharge (UHF PD) signal in gas insulated switchgear (GIS) at different scales. The trend curves of complex wavelet transform energy entropy (CWT-EE) under different decomposition scale were analyzed, and it was found that the PD feature information mainly distributed in the scales, in which the gradient of CWT-EE is big. Besides, The CWT-EE characteristics and their scales were extracted to the structure characteristic pairs for PD type identification. The recognition results show that the characteristic pair could effectively identify four typical defects in GIS and obviously reduce the feature dimension.
\end{abstract}

\section{Introduction}

The partial discharge (PD) detection is an important field in gas insulated switchgear (GIS) live detection [1]. The insulation defects and hidden dangers of GIS can be found by PD detection in early time. The method of Wavelet analysis is widely used for PD detection and analysis, and the statistical characteristics of wavelet coefficients, wavelet energy characteristics and singular value characteristics of PD signals are used for PD identification [2-5]. However, optimization of decomposition scale of PD signal under different defects are always ignored when processing the PD signal by means of wavelet analysis. For example, either do they select a decomposition scale for wavelet decomposition directly, or regard it as the same optimal decomposition layer number under default conditions in different defects [6]. In fact, the spectrum distribution is different under different defects and different discharge severity under the same defect. As a result, PD signal energy spectrum distribution characteristics are different under different defects and discharge severity, and the optimal decomposition scale of PD signal is also different. In addition, the extraction and selection of feature parameters are based on the difference of the size and distribution of feature values, while the scale and location information of the distribution of feature parameters are not taken into account [6-7].

In this paper, complex wavelet transform (CWT) is applied to decompose ultra-high frequency (UHF) PD signals at different scales, and the characteristics of complex wavelet energy entropy (CWT-EE) are constructed. The variation rule of CWT-EE with decomposition scale and discharge voltage is analyzed, and it is found that PD signal characteristic information is mainly distributed in several scales with large change gradient of CWT-EE, and PD signal characteristic changes are different under different discharge voltage for different defects or the same defect. Therefore, this paper extracts the maximum energy entropy of CWT-EE gradient and its corresponding decomposition scale location, and constructs the scale parameters-energy entropy characteristic pairs (SP-EE) of complex wavelet for PD type recognition. The results show that SP-EE features can effectively identify four typical defects in GIS and significantly reduce the feature dimension.

\section{Identification of partial discharge defect}

There are four common defects in GIS [10], which are air gap defect (G(gap)), insulator surface metal contamination defect $(\mathrm{M}(\mathrm{metal}))$, metal protrusion defect $(\mathrm{N}($ needle $))$ and free metal particle defect $(\mathrm{P}($ particle $))$. In this paper, four defect models are designed according to the four defect characteristics, as shown in figure 1 . 


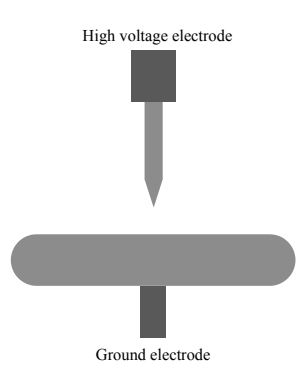

(a)

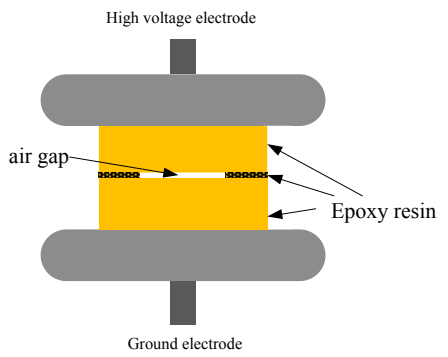

(c)

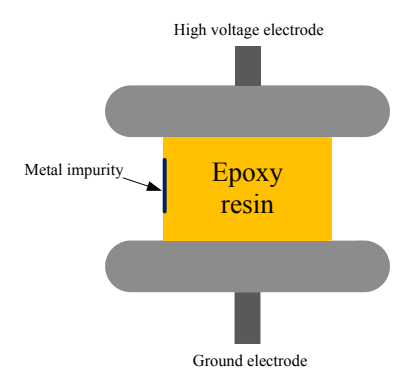

(b)

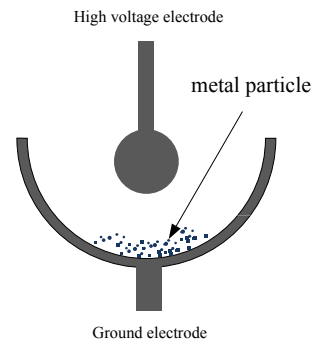

(d)

Figure1. The structure of insulation fault models. (a) metal protrusion defect. (b) metal contamination defect. (c) air gap defect. (d) free metal particle defect

In order to capture the PD signal of defect model, the experimental circuit is set up, as shown in figure 2. A Tektronix7100 oscilloscope with a maximum sampling rate of $20 \mathrm{GS} / \mathrm{s}$ and storage depth of $48 \mathrm{MB}$ is utilized. Also a UHF sensor with $200 \sim 800 \mathrm{MHz}$ bandwidth is configurated.

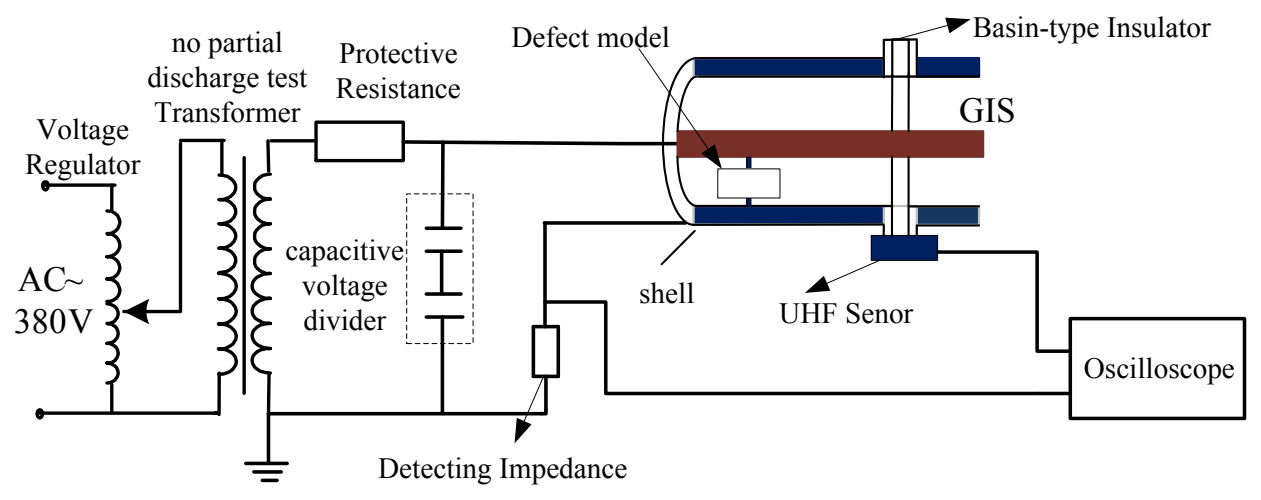

Figure 2. Schematic diagram of PD experiment

In the experiment, four kinds of artificial defects were placed on $126 \mathrm{kV}$ GIS partial discharge experiment simulation platform and filled with $0.4 \mathrm{mpa}$ SF6 gas. The sampling rate was set at $5 \mathrm{Gs} / \mathrm{s}$, and the signal length was set at 50,000 sampling points. UHF PD signals of four kinds of defects at different voltages (as shown in table 1) were collected respectively. 100 groups of effective discharge samples were collected at each discharge voltage, and a total of 400 groups of PD sample data of four kinds of defects were obtained. Figure 3 shows the UHF PD signals of the four defect models.

Table1. Experimental voltages

\begin{tabular}{|c|c|}
\hline Defect Type & Test Voltage $/ \mathrm{kV}$ \\
\hline $\mathrm{N}$ & $9.3 、 15.1 、 19.5,26.3$ \\
\hline $\mathrm{P}$ & $11 、 13.7 、 16.8 、 20.6$ \\
\hline M & $8.3 、 11.6,15.4 、 17.5$ \\
\hline G & $10 、 13.5 、 16.5 、 21$ \\
\hline
\end{tabular}

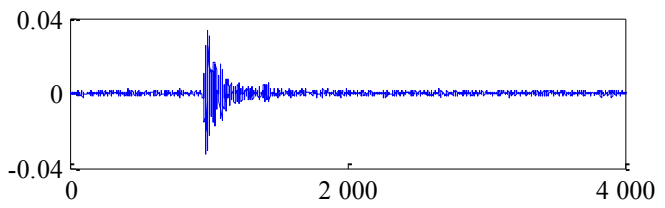

(a)

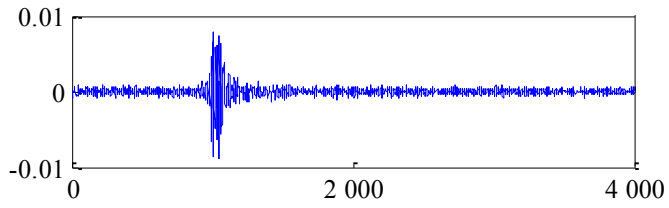

(b)

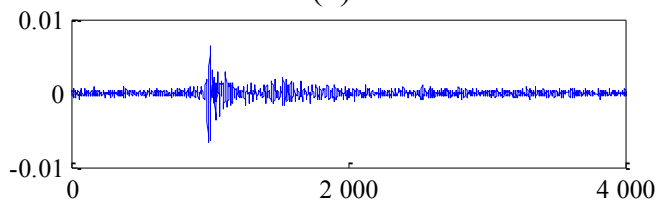

(c) 


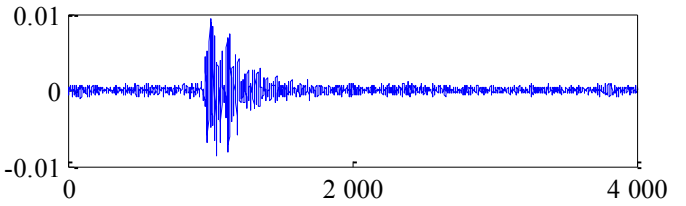

(d)

Figure 3. PD signals of the four typical insulation defects. (a) N-type defect. (b) P-type defect. (c) M-type defect. (d) G-type defect

\section{Analysis of complex wavelet energy entropy}
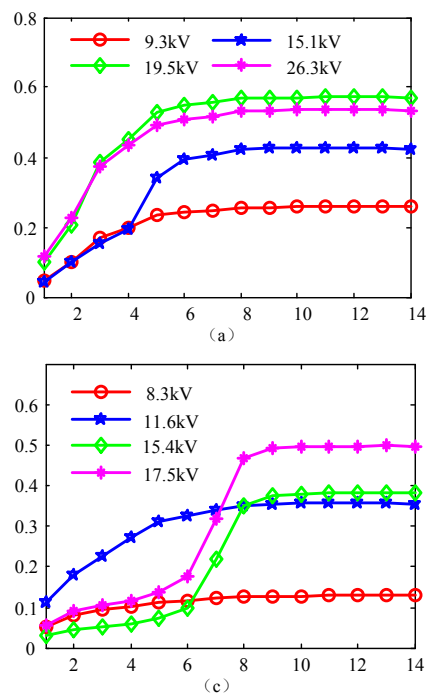

Figure 4. Curves of CWT-EE vs. decomposition scale under different experimental voltages.

(a) N-type defect. (b) P-type defect. (c) M-type defect. (d) G-type defect

Figure 4 shows the CWT-EE curve that four typical pd signals in the gis are decomposed from 0 to 14 layer. It can be found that the PD signals under the four defects are saturated with the increase of the number of decomposition layers, but the growth trend are different. It can be seen from Fig. 4 (a) that Under $\mathrm{N}$ defects, the higher the voltage, the greater the CWT-EE. And when the number of decomposition layer is six, CWT-EE saturates, the process of the CWT-EE is more comprehensive. As shown in Fig. 4(b), the CWT-EE size and the change curve are the same in the small discharge voltage. However, the discharge gradually strengthened as the voltage increased, and the CWT-EE changes as a phenomenon that is rapid slow - rapid - saturation. According to Fig. 4(c), the growth of CWT-EE is relatively flat as layer increases in the small discharge voltage. And when the discharge voltage is $11.6 \mathrm{kV}$, the growth of CWT-EE is faster between 1 layer and 6 layer, and it is saturated after the sixth layer. When the discharge voltage increases to $15.4 \mathrm{kV}$, a S-shape increasing curve is generated as the number of decomposition layers increases. When the layer is contained between 6 layer and 8 layer, it is greatly increased, and tends to be saturated after the 8th layer. Fig. 4 (d) shows that the growth law of CWT-EE is same in different experimental voltages for $\mathrm{G}$ defect. It all changes from fast growth to low growth, and grow fast again up to saturation. Also the discharge voltage has little effect on the distribution of CWT-EE.

\subsection{The change curve of the wavelet energy entropy under different discharge voltage}

In this paper, the number of PD sampling point is set to 50,000 , according to the CWT binary principle, we select decomposition level as 14 layers. In order to eliminate the influence of random factors on the result analysis, $50 \mathrm{PD}$ sample signals under each voltage and each defect are randomly selected. Meanwhile, every PD signal is processed by method of normalization in order to extract each PD signal in different voltages, and the average of the PD signal is measured in different decomposition scale.
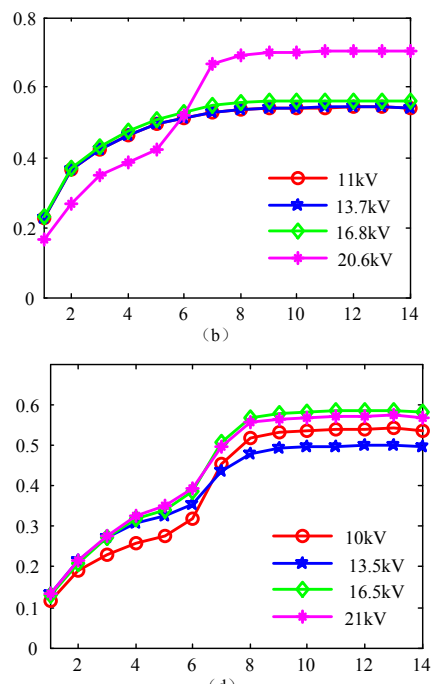

\subsection{Trend analysis of wavelet energy entropy}

Shannon entropy of the $\mathrm{k}$ series and $\mathrm{m}$ layer after a high frequency decomposition can be expressed as [8-9]

$$
H(k)=-\frac{\left(W T_{\mathrm{M}(m, k)}\right)^{2}}{\sum_{j=1}^{j=L_{m}}\left(W T_{\mathrm{M}(m, j)}\right)^{2}} \bullet \lg \left(\frac{\left(W T_{\mathrm{M}(m, k)}\right)^{2}}{\sum_{j=1}^{j=L_{m}}\left(W T_{\mathrm{M}(m, j)}\right)^{2}}\right)
$$

According to (1), a function is constructed that $f(x)=-x \lg x$, so the derived function of $f(x)$ is

$$
f^{\prime}(x)=-\lg x-\lg e
$$

It can be seen that $f(x)$ is monotone increasing function in the $\left(0, e^{-1}\right]$ range and be monotone decreasing in the $\left[\mathrm{e}^{-1},+\infty\right)$ range. Therefore, for PD signal under the decomposition layer, the larger the signal energy, the more disordered the energy distribution. And the proportion of energy contained in a single sampling point to the total energy at the corresponding scale is smaller. That means the $\mathrm{x}$ is will be smaller. So, the CWT-EE will be greater as a whole.

The physical significance of multi-scale decomposition of complex wavelet is to decompose PD signal in time domain and frequency domain simultaneously. The high frequency signal at different 
scales represents the distribution of signal energy in different frequency domain space. As be shown in Fig. 4, the difference of CWT-EE between different scales is the incremental amount of information generated by increasing the number of decomposition layers.

Fig. 5 shows the reconstructed signal diagram and spectrum diagram of PD signals with N-type defects after decomposition of CWT at 14 layers, in which the vertical coordinate is amplitude $/ \mathrm{mV}$. It can be found that only weak PD pulse signals can be seen at the first layer of the reconstructed signals, most of which are high-frequency interference signals, while obvious PD pulse signals cannot be distinguished above the eighth layer. PD pulse signals are mainly distributed at the second to seventh layer. Compared to Fig. 4(a), it can be seen that the fast growth interval of CWT-EE is layer $1 \sim 7$, which can see the scale of PD signal pulse obviously.

Fig. 4 and Fig. 5 show that the variation law of CWTEE curve for N-type defects under different voltages is basically similar, because the local electric field distortion caused by $\mathrm{N}$-type defects is relatively stable, also the
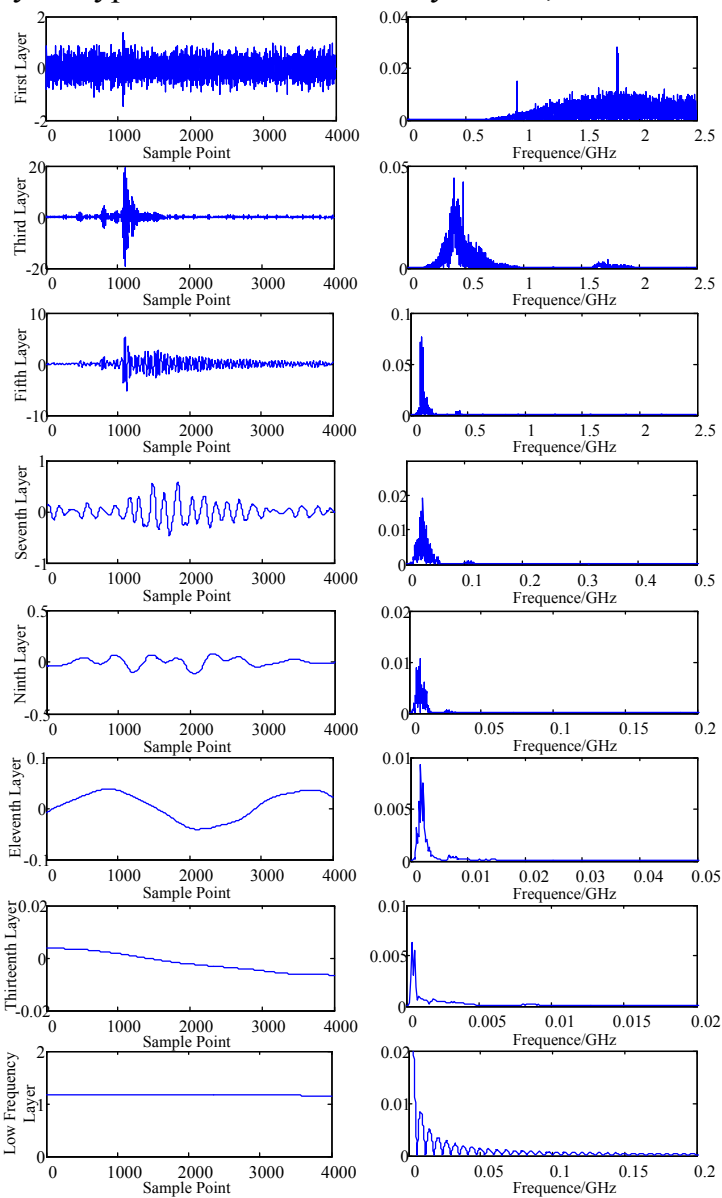

Figure.5 The restructure PD signals of each decompose level and its spectrum under needle defect after decomposed 14 layer

The energy entropy difference at adjacent decomposition scales can be expressed as following equaion.

$$
\Delta E(J)=E_{\text {Total }}(J)-E_{\text {Total }}(J-1), 2 \leq J \leq 14
$$

Characteristic pairs $\Theta$ is constructed as

$$
\Theta=\{J, \Delta E(J)\}, \quad 2 \leq J \leq 14
$$
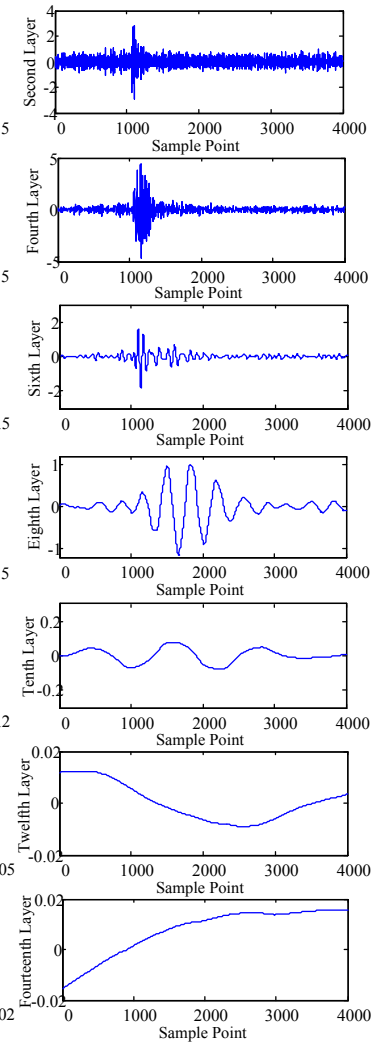

discharge process is. For G-type, M-type and P-type defects, the distribution of available characteristic information is the same as N-type defects, that is, the istribution is at the scale where CWT-EE grows rapidly. PD signal features of different defects, which can be used

\subsection{Extracting scale parameters-energy entropy}

According to Fig. 4, the energy entropy is different at different decomposition scales and different discharge voltage for the same defect, and the energy entropy difference at adjacent decomposition scales also has regularity. After decomposition of $J$ layer, the total CWT-

$$
E_{\text {Total }}(J)=\sum_{m=1}^{m=J} E(m)
$$
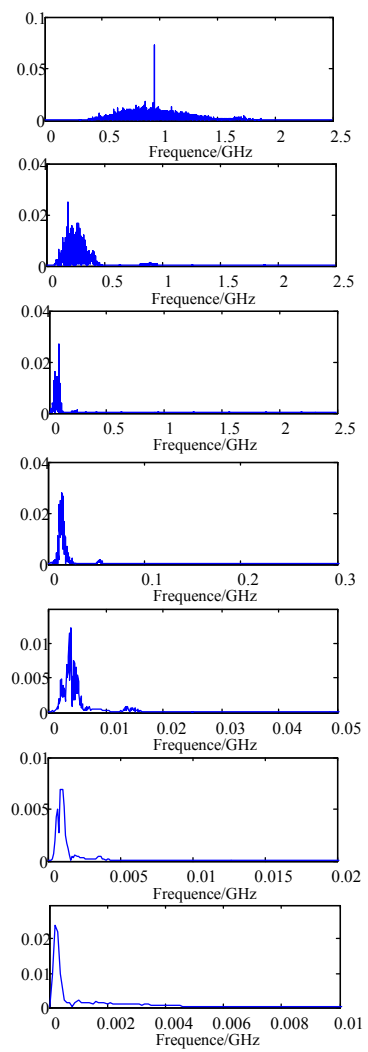
contain both scale information and energy information, which can solve the problem of feature extraction methods that lost the wavelet scale information. In this paper, the three maximum energy entropy of CWT-EE and its corresponding scale are extracted as the characteristic information. Fig.6. shows the extracted characteristic pairs of CWT-EE, in which the abscissa axis represents the 
decomposition scale, and the ordinate axis is the energy entropy and frequency.

From Fig. 6, it can be found that the three largest CWT-EE of different defects have different sizes and values, and their scales are also different. The three largest decomposition scales of CWT-EE for N-type defects are mainly: scale 1, 2 and 4; G-type defects are mainly distributed at scale 1,2 and 3; M- type defects are mainly distributed at scale 1, 2 and 6; and G-type defects are mainly distributed at scale 1,2 and 6. Meanwhile, three

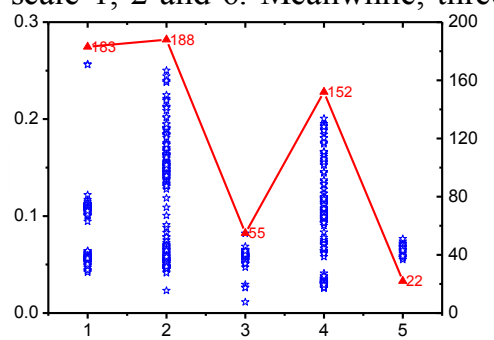

(a)

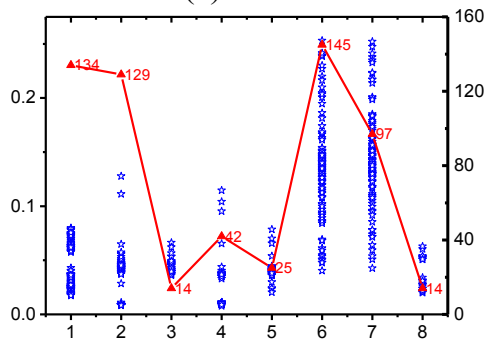

(c) major distribution characteristics of decomposition scale is also different for different defects. N-type, P-type and G-type defects under scale characteristics is concentrated. $\mathrm{N}$-type defects with probability of $87.17 \%$ are distributed at scale 1, 2 and 4; P-type defects with probability of $89 \%$ are distributed at scale 1, 2 and 3; G-type defects with probability of $82.17 \%$ probability are distributed at scale 1, 2 and 6. And M-type defect is relatively scattered, it is distributed at scale 1, 2 and 6 with a probability of $68 \%$.

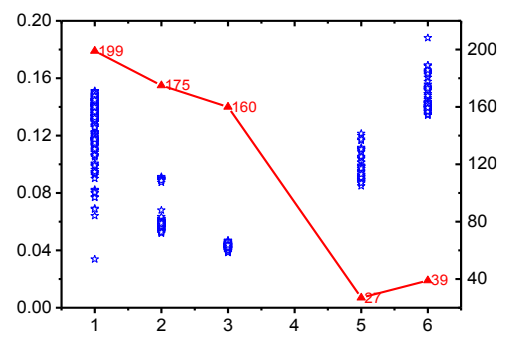

(b)

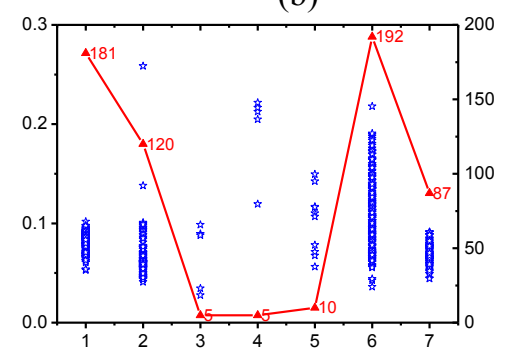

(d)

Figure.6 Numerical distributions map of SP-EE. (a) N-type defect. (b) P-type defect. (c) M-type defect. (d) G-type defect

In addition, according to Fig. 6, PD signals with different defects are decomposed at different scales, the CWT-EE values and distribution interval widths are also different. For N-type defects, the width of CWT-EE distribution interval at the 2 nd and 4 th decomposition scale is significantly larger than that at the $1 \mathrm{st}$ decomposition scale. For P-type defects, the width of CWT-EE distribution range shows a decreasing trend at the 1 st $\sim 3$ rd scale, and the energy entropy also decreases gradually. The distribution interval of CWT-EE for Mtype defects is narrow at scale 1 and 2, but is broad at scale 6 and 7. The G-type defects is same as M-type defects at scale 1 and 2, but is just broad at scale 6 . It also can be seen that the size of CWT-EE varies with different defects and different scales. For example, the the size of CWT-EE is relatively large at scale 2,1,6-7 and 6 for N-type, P-type, M-type and G-type respectively.

In a word, it can be seen that the characteristics of different discharge signals can be effectively represented by constructing SP-EE characteristic pairs while retaining the energy entropy characteristics and corresponding scale characteristics.

\section{Defect identification}

Support vector machine (SVM) is a machine learning theory based on the VC theory and SRM principle of statistical learning theory. It is especially suitable for solving the problems of pattern classification and regression analysis in the case of high dimensional nonlinearity [11]. In this paper, a one-to-one SVM was selected to identify UHF PD signals for four typical defects, and radial basis function (RBF) was selected as the kernel function of the SVM. RBF function takes system default value. We randomly select 200 groups of sample data for training, which come from the characteristic database of each type defect. The results can be seen from the Table 2 .

For comparison, CWT-EE characteristic after 8-layer decomposition are also adopted for PD identification in this paper. As is shown in Table 2, SP-EE energy characteristic have higher recognition rate than CWT-EE energy, and the overall recognition rate reaches $95 \%$. For G-type defects, although the recognition rate increases slightly when PD is identified by using CWT-EE characteristics. In addition, CWT-EE characteristics can significantly reduce the recognition rate of M-type defects, which is less than $90 \%$. Meanwhile, only 6 characteristics were adopted for SP-EE characteristic pairs, while CWTEE selects 9 characteristic, which include 8 highfrequency layer characteristics and 1 low-frequency layer characteristic. So, the SP-EE is more effectively.

If the CWT-EE characteristic sequence is regarded as a one-dimensional information, then the SP-EE characteristic pair is a two-dimensional information. The SP-EE characteristic pair not only retains the energy entropy feature information, but also retains the location information of the characteristic pair.

In this way, PD signal characteristics can be more effectively represented. Therefore, PD identification rate 
can be significantly improved by capturing SP-EE characteristic pairs.

Table 2. The recognition accuracy of UHF PD signal

\begin{tabular}{c|cc}
\hline $\begin{array}{c}\text { Defect } \\
\text { Type }\end{array}$ & SP-EE & CWT-EE \\
\hline $\mathrm{G}$ & $92.00 \%$ & $93.00 \%$ \\
$\mathrm{M}$ & $91.50 \%$ & $86.00 \%$ \\
$\mathrm{~N}$ & $96.50 \%$ & $92.50 \%$ \\
$\mathrm{P}$ & $100.00 \%$ & $95.00 \%$ \\
\hline Average & $95.00 \%$ & $91.675 \%$ \\
Value & &
\end{tabular}

\section{Conclusion}

The complex wavelet transform was used to process the ultra-high frequency partial discharge signal in gas insulated switchgear at different scales in this paper. Through the experiment we see that PD information of CWT-EE under different defects has different characteristics with the change of decomposition scale. The difference of PD signal CWT-EE variation trend under different defects lies in the difference of PD signal spectrum distribution. CWT-EE with fast change scale can characterize PD signal characteristics under different defects and it can be used for PD identification. Moreover, SP-EE characteristic pairs retain both the signal complexity information and location information of PD signal layers, and have better discrimination sensitivity and better classification effect than CWT-EE energy feature.

\section{References}

1. SCHAIK N V, CZASZEJKO T. Conditions of discharge-free operation of XLPE insulated power cable systems [J]. IEEE Transactions on Dielectrics and Electrical Insulation, 2008, 15(4): 1120-1130

2. Si Wenrong, Li Junhao, Li Dajian, et al. Fast feature extraction technique for PD pulse sha pebased on wide band detection [J]. Advanced Technology of Electrical Engineering and Energy, 2008, 27(2): 2125+76.

3. Zhao Feng, Li Shuo. Fault diagnosis in transformer based on entropy weighted. [J]. Advanced Technology of Electrical Engineering and Energy, 2015, 34(1): 57-61.

4. H.-C. Chen. Fractal features-based pattern recognition of partial discharge in XLPE power cables using extension method[J]. IET Generation, Transmission \& Distribution, 2012, 6(11):1096-1103

5. LI Yanran, QIAN Yong, CHEN Xiaoxin, et al. A Study of Weibull Distribution Characteristics for Partial Cable Discharging [J]. Electrical Automation, 2015, 5: 111-114.

6. TANG Ju, DONG Yulin, FAN Lei, et al. Feature information extraction of partial discharge signal with complex wavelet transform and singular value decomposition based on Hankel matrix [J]. Proceedings of the CSEE, 2015, 35(7): 1808-1817.

7. Yang Zhichao, Fan Lixin, Yang Chengshun, et al. Partial discharge pattern recognition based on auto regressionmodel and hypersphere support vector machine [J]. Advanced Technology of Electrical Engineering and Energy, 2015, 35(9): 29-34.

8. Cifman R R, Wickerhauser M V. Entropy based algorithms for best basis selection[J]. IEEE Transaction on Information Theory, 1992, 38(2): 713718 .

9. ZHANG Guangdong, QIN Rui, ZHANG Zhongyuan, et al. Extraction and analysis of characteristic spectrum of partial discharge in GIS based on UHF method [J]. High Voltage Apparatus, 2016, 52(9): 7177.

10. TANG Ju, LIN Junyi, ZHUO Ran, et al. Partial discharge type recognition based on support vector data description [J]. High Voltage Engineering, 2013, 39(5): 1046-1053

11. [11] C. Cortes, V. Vapnik, "Support-vector networks", Machine learning, Vol. 20, No. 3, pp. 273297, 1995 